\title{
The hyh mutation uncovers roles for $\alpha$ Snap in apical protein localization and control of neural cell fate
}

\author{
Teresa H Chae ${ }^{1,3}$, Seonhee Kim ${ }^{1,3}$, Karla E Marz ${ }^{2}$, Phyllis I Hanson ${ }^{2}$ \& Christopher A Walsh ${ }^{1}$
}

\begin{abstract}
The hyh (hydrocephalus with hop gait) mouse shows a markedly small cerebral cortex at birth and dies postnatally from progressive enlargement of the ventricular system ${ }^{1,2}$. Here we show that the small hy $h$ cortex reflects altered cell fate. Neural progenitor cells withdraw prematurely from the cell cycle, producing more early-born, deep-layer cerebral cortical neurons but depleting the cortical progenitor pool, such that late-born, upper-layer cortical neurons are underproduced, creating a small cortex. hy $h$ mice carry a hypomorphic missense mutation in the gene Napa encoding soluble N-ethylmaleimidesensitive factor (NSF) attachment protein alpha ( $\alpha$ Snap), involved in SNAP receptor (SNARE)-mediated vesicle fusion in many cellular contexts. A targeted null Napa mutation is embryonically lethal. Altered neural cell fate is accompanied by abnormal localization of many apical proteins implicated in regulation of neural cell fate, including $\mathrm{E}$-cadherin, $\beta$-catenin, atypical protein kinase $\mathrm{C}$ ( $\mathrm{aPKC}$ ) and INADL (inactivation-noafterpotential D-like, also known as protein associated with Lin7, or Pals1). Apical localization of the SNARE Vamp7 is also disrupted. Thus, $\alpha$ Snap is essential for apical protein localization and cell fate determination in neuroepithelial cells.
\end{abstract}

We mapped hyh on Chromosome 7 (ref. 2) to a 0.3 -cM region spanned by two BACs using serial rounds of sequence-tagged site (STS) cloning, generation of new polymorphic markers and analysis of 1,657 meioses (Fig. 1a). Our own BAC sequencing and publicly available mouse and human genome sequence data identified five genes in this interval. We found a mutation only in Napa, which encodes $\alpha$ Snap. Northern-blot analysis showed low levels of Napa RNA in hyh mice (Fig. 1b), although the sizes of hybridizing RNAs were normal, suggestive of mRNA instability. Western-blot analysis showed low levels of $\alpha$ Snap (Fig. 1c). Sequence analysis of Napa cDNA and genomic DNA showed a $\mathrm{G} \rightarrow \mathrm{A}$ missense mutation in exon 4 that results in the amino acid substitution M105I (Fig. 1d). This sequence change was not present in other mouse strains (B6, B10, C3H), including the ancestral strain from which hyh arose (data not shown). Met105 is conserved in all known mammalian $\alpha$ Snap proteins (human, mouse, rat, pig, cow), but not in the yeast Saccharomyces cerevisiae, and M105I does not change amino acid charge, suggesting that the small amount of $\alpha$ Snap in the mutant may have residual function.

Two overlapping BACs encoding Napa rescued the hyh phenotype (Fig. 1a). We mated stable transgenic lines carrying each $B A C$ to create homozygous hyh mice carrying each BAC transgene and littermate homozygous hyh mice without the transgene. Although the hyh phenotype was fully penetrant on the hybrid background of this cross (Fig. 1e), homozygous hyh mice carrying either BAC transgene lacked characteristic central nervous system abnormalities of $h y h$ mutants.

To determine the effects of the $h y h$ mutation on $\alpha$ Snap, we purified protein containing the M105I mutation and analyzed $\alpha$ Snap in vitro. Met105 is exposed on the 'back' side of $\alpha$ Snap opposite those residues that alter SNARE complex binding ${ }^{3}$ (Fig. 2a). Far ultraviolet circular dichroism spectra of mutant and wild-type proteins were similar (Fig. $2 \mathbf{b}$ ), suggesting that this mutation does not substantially alter protein folding. Consistent with this, expression of Napa cDNA encoding the M105I mutation in Cos7 cells produced normal levels of protein (data not shown), suggesting that the low levels of $\alpha$ Snap in $h y h$ mice reflects mRNA instability and not protein instability. Fluorescence resonance energy transfer (FRET) analysis of $\alpha$ Snap binding to synaptic SNARE complexes showed that binding of M105I mutant protein was indistinguishable from that of wild-type $\alpha$ Snap (Fig. 2c), and NSF-mediated SNARE complex dissociation was comparable in the presence of M105I mutant or wild-type $\alpha$ Snap (Fig. 2d). Therefore, we conclude that $h y h$ is a hypomorphic mutation that results in low levels of protein owing to an unstable mRNA and not to disrupted protein function per se. It is formally possible, however, that the M105I mutant may be less functional than normal in processes that we have not directly tested. The residual protein in hyh mutants must be partially functional, because a targeted null mutation in Napa is embryonically lethal in mice (Supplementary Fig. 1 and Supplementary Methods online), consistent with results of RNA interference studies in Caenorhabditis elegans that suggest lethality at the two-cell stage ${ }^{4}$.

The cerebral cortex in hyh embryos was substantially smaller than in wild-type embryos. The mutant forebrain was distinguishable as early as embryonic day (E) 12.5 only by a widened roof plate

${ }^{1}$ Howard Hughes Medical Institute, Beth Israel Deaconess Medical Center and Department of Neurology and Program in Neuroscience, Harvard Medical School, HIM 816, 4 Blackfan Circle, Boston, Massachusetts 02115, USA. ${ }^{2}$ Department of Cell Biology and Physiology, Washington University School of Medicine, St. Louis, Missouri 63110, USA. ${ }^{3}$ These authors contributed equally to this work. Correspondence should be addressed to C.A.W. (cwalsh@bidmc.harvard.edu).

Published online 1 February 2004; doi:10.1038/ng1302 
a
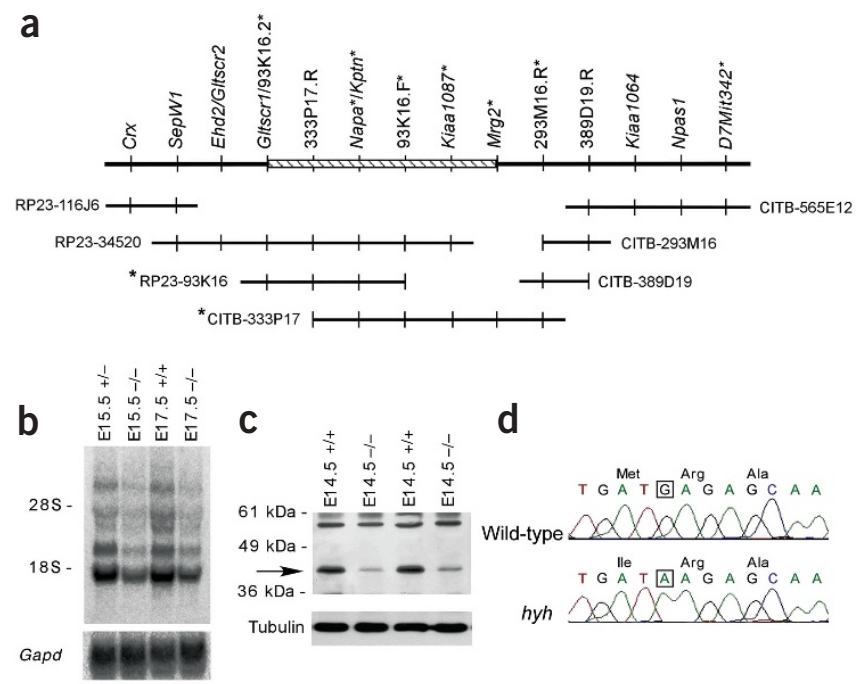

e

\begin{tabular}{|c|c|c|c|c|}
\hline BAC & $\begin{array}{l}\text { Percent } \\
\text { expressing } \\
\text { hyh phenotype }\end{array}$ & $\begin{array}{l}\text { Percent pheno- } \\
\text { typically normal } \\
\text { transgenic mice }\end{array}$ & $\begin{array}{l}\text { Number of } \\
\text { transgenic } h y / \\
\text { mice analyzed }\end{array}$ & $\begin{array}{l}\text { Number of } \\
\text { independent } \\
\text { transgenic lines }\end{array}$ \\
\hline None & $100 \%$ & N/A & $5^{*}$ & N/A \\
\hline $93 \mathrm{~K} 16$ & 0 & $100 \%$ & 17 & 3 \\
\hline $333 \mathrm{P} 17$ & 0 & $100 \%$ & 6 & 1 \\
\hline
\end{tabular}

(Fig. 3a,e). By E14.5, the cerebral cortex was smaller and the third ventricle was displaced dorsally in $h y h$ mutants (Fig. $\mathbf{3 b}, \mathbf{f}$ ), and by E16.5 (Fig. 3c,g) and postnatal day 0 (P0; Fig. 3d), the cortex was markedly smaller in hyh mutants compared with wild-type embryos. hyh mutants also had heterotopic neurons in the ventricular and marginal zones (Fig. 3g,h) and a small, malformed hippocampus (data not shown). Progressive hydrocephalus distorts the brain and leads to death a few weeks postnatally ${ }^{1}$. Despite the small cortex, forebrain patterning was unexpectedly normal (Supplementary Fig. 2 and Supplementary Methods online).

a

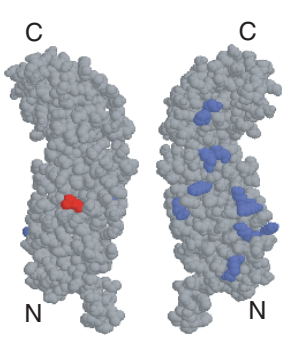

C

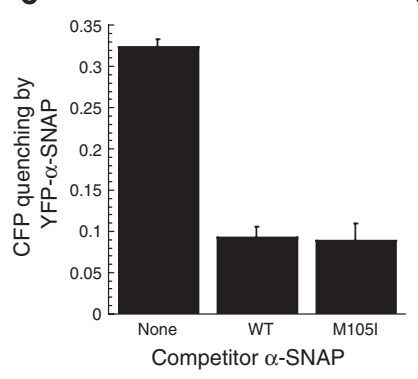

b

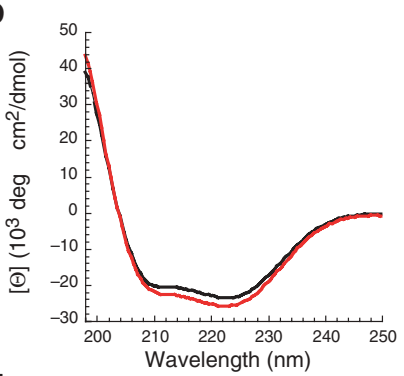

d

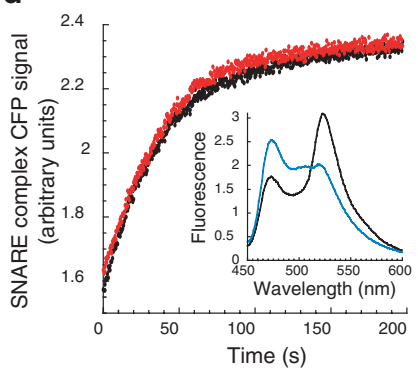

Figure 1 Positional identification of the hyh mutation. (a) Physical map of the region containing Napa bounded proximally by Crx and distally by D7Mit342. A BAC contig spanning the hyh locus is illustrated below. Genotyping of recombinant mice at newly developed polymorphic markers (indicated by asterisks) allowed the refinement of the hyh candidate interval (indicated by a hatched box) to the region bounded proximally by $93 \mathrm{~K} 16.2$ and distally by Mrg2. This region contains five genes, Gltscr1, Napa, Kptn, Kiaa1087 and Mrg2, and is spanned by just two overlapping BACs, 93K16 and 333P17. BACs isolated from the CITB mouse BAC library are designated by CITB and those isolated from the RPCI-23 mouse BAC library are designated by RP23 followed by the clone address. STSs contained in a gene are indicated by the italicized gene symbol. (b) Northern-blot analysis of Napa in hyh mutants. Total RNA from brain tissue of wild-type $(+/+)$, heterozygous (+/ -$)$ and homozygous hyh (-l-) embryos at E15.5 and E17.5 was hybridized to a Napa probe, showing less expression of Napa in hyh mutants with preservation of transcript sizes. The same blot was stripped and probed for Gapd expression as a loading control (lower panel). (c) Western-blot analysis of $\alpha$ Snap in hyh mutants. Whole-brain lysates from E14.5 wildtype (+/+) and hyh mutant (-/-) embryos were probed with a monoclonal antibody to $\alpha$ Snap. $\alpha$ Snap protein levels (arrow) were substantially lower in hyh mutants. The same blot was stripped and probed with an antibody to tubulin as a loading control (lower panel). (d) Sequence analysis of Napa in hyh mutants. The $\mathrm{G} \rightarrow \mathrm{A}$ missense mutation (boxed nucleotides) in exon 4 changes Met105 to Ile. (e) The effects of BAC rescue on the hyh phenotype. The BACs used are shown in a. In addition to the mice listed, six additional hyh/hyh mice lacking a BAC transgene died after P21, probably owing to expression of the hyh phenotype, before phenotypic analysis was possible.

The small cerebral cortex in hyh mutants was associated with alteration in neuronal cell fate, detectable using markers for specific neuronal types. At P3, after neurogenesis is complete, the cortex of hyh mutants had excessive early-born neurons and few late-born neurons. Immunoreactivity to Reelin, which marks some of the earliest-born neurons of the cortical marginal zone ${ }^{5}$, was greater, as was the thickness of the marginal zone itself (Fig. 3ij,j). Similarly, immunoreactivity to Foxp2, which marks early-born neurons in layer VI (ref. 6), was greater, both in density of Foxp2-positive cells in layer VI and in the proportion of the cortical thickness containing Foxp2-immunoreactive neurons
Figure $2 \mathrm{M} 105 \mathrm{I} \alpha \mathrm{Snap}$ is indistinguishable from wild-type $\alpha$ Snap in vitro. (a) Homology model of $\alpha$ Snap, based on the crystal structure of yeast Sec17p, showing the surface on which Met105 (red) is exposed and the opposite surface on which residues involved in interaction with the SNARE complex (blue) are located ${ }^{3}$. (b) Far ultraviolet circular dichroism spectra of wild-type $\alpha$ Snap (black) and M105I $\alpha$ Snap (red). The helical content of each is within $5 \%$ of that predicted from the homology model. (c) Binding of wild-type (WT) and M105I $\alpha$ Snap to synaptic SNARE complex. Shown is a competition-based binding assay in which nothing (left), unlabeled wild-type $\alpha$ Snap ( $8 \mathrm{mM}$, middle) or M105I $\alpha$ Snap ( $8 \mathrm{mM}$, right) competes with $2 \mathrm{mM}$ YFP- $\alpha$ Snap for binding to SNARE complex containing syntaxin1A-CFP. CFP quenching indicates binding of YFP- $\alpha$ Snap to the SNARE complex and was reduced by the binding of unlabeled $\alpha$ Snap. Values shown are mean \pm s.d. $(n=3)$. (d) Time course of SNARE complex disassembly mediated by NSF and either wild-type $\alpha$ Snap (black) or M105I $\alpha$ Snap (red). SNARE complex containing syntaxin 1A-CFP and synaptobrevin-YFP was incubated with $\alpha$ Snap, followed by addition of NSF to initiate SNARE complex disassembly. Disassembly is seen as an increase in CFP emission (472 nm), which occurs when the distance between CFP and YFP tags attached to SNAREs increases beyond the range in which fluorescence resonance energy can be transferred from CFP to YFP. The inset shows the fluorescence spectra of SNARE complex before (black) and after (blue) SNARE complex disassembly when excited at $434 \mathrm{~nm}$. 
(Fig. 3k,l). In contrast, later-born neurons of layers II-IV, which are immunoreactive for Cux-1 and Brn-1 (ref. 7), were much less abundant in cortex of hyh mutants (Fig. 3m,n and data not shown), consistent with the overall reduction in cortical size. These data suggest that the Napa mutation alters the balance of early-born and late-born neurons, shifting the laminar organization of the resulting cortex.

We observed alterations in cortical cell fate reflecting precocious generation of neurons and premature depletion of progenitor cells in the cortical neuroepithelium in 14 hyh homozygotes examined at ages E12-P3. Immunostaining for Nestin, a marker of cortical progenitor cells, indicated progressive loss of progenitor cells in hyh mutants from E12.5 (Fig. 4a,b), early in neurogenesis, through later stages (Fig. 4c,d). Loss of progenitor cells in cortex of hyh mutants was confirmed by staining with antisera to RC-2, Ki67 and phosphorylated histone $\mathrm{H} 3$, which label dividing cells (data not shown). Analysis of incorporation of 5-bromodeoxyuridine after a 30-min pulse showed similar proportions of dividing cells in hyh and wild-type embryos at E14 (data not shown), suggesting that the loss of progenitors does not reflect gross alteration of cell cycle kinetics but may instead result from another mechanism, such as altered cell fates. The dwindling progenitor pool in hyh mutants was associated with excessive formation of neurons at early developmental stages. Immunoreactivity to chondroitin sulfate proteoglycan (CSPG) at E14.5, which marks early-born neurons of the preplate ${ }^{8}$, was greater in $h y h$ mutants than in wild-type embryos (Fig. 4e,f), confirming excessive early-born neurons as indicated by Reelin immunostaining. Immunostaining for Dcx, TuJ1 and Vamp2, markers of postmitotic neurons ${ }^{9}$, confirmed excessive early generation of neurons in hyh mutants at E12-E16 (Fig. 4g-r and data not shown) and corresponding reduction in the progenitor zone at E16.5 (Fig. 4o-r). Glial fibrillary acidic protein analysis showed no obvious evidence of precocious gliogenesis. Neural progenitor cells normally divide to generate either two proliferative daughter cells or one or two postmitotic neuronal daughter cells, with 'proliferative' divisions predominating early and neuron-generating divisions predominating later ${ }^{10-13}$. Our data suggest that early overproduction of neurons at the expense of progenitor cells causes depletion of progenitors and suggest a role for $\alpha$ Snap in regulating cell fate.

As $\alpha$ Snap regulates protein trafficking ${ }^{14,15}$, it could regulate cell fate by controlling polarized protein transport in neural progenitors. We found that hyh mutants showed marked abnormalities of the normally polarized structure of the ventricular zone. Confocal analysis of $\mathrm{F}$-actin distribution in the ventricular zone (stained with rhodamine-phalloidin) showed that some adherens junctions were displaced away from the ventricular surface and not as regularly aligned at the ventricular surface in hyh mutants (Fig. 5a,b). Analysis of $\alpha$-catenin, which links F-actin to adherens junctions, yielded similar observations (data not shown). Distribution of Ecadherin, a primary membrane component of adherens junctions

\begin{abstract}
Figure 3 Abnormal cell fates in cerebral cortex of hyh mutants. (a-h) Coronal sections of the forebrain of age-matched normal $(\mathbf{a}-\mathbf{d})$ and hyh mutant (e-h) embryos show that the hyh phenotype is noticeable as early as E12.5 (a,e) by the broadening of the interhemispheric sulcus (arrow). The size of the cerebral hemispheres and other regions of the forebrain was otherwise indistinguishable between mutants and wild-type embryos at this age. By E14.5, the cerebral hemispheres of hyh mutants were smaller than normal, with increased dorsal midline structure (arrow) relative to the cortex, so that the midline third ventricular roof separated the two hemispheres to a greater extent than in controls $(\mathbf{b}, \mathbf{f})$. The small size of the cerebral hemispheres was also evident by comparisons at E16.5 (c,g), and the roof of the third ventricle also showed an excess of papillary epithelium that was differentiating into choroid plexus (arrow). At this age, there were also laminar disruptions of the cerebral cortex (arrowhead) and areas of denudation of the ventricular lining of the ventral third ventricle (asterisks). At PO (d,h), the dorsal midline of the third ventricle, composed of
\end{abstract} choroid plexus epithelium, continued to protrude
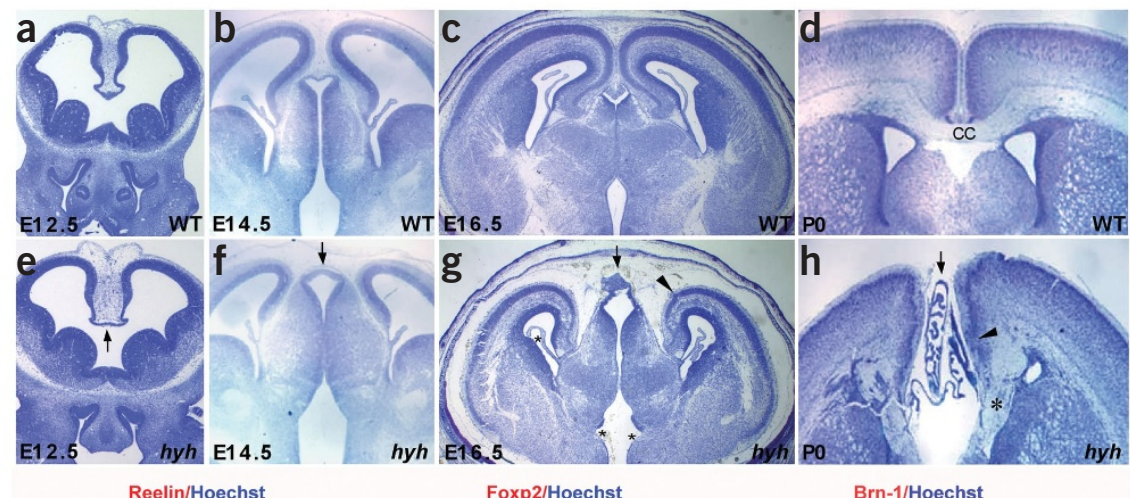
$\mathrm{h}$

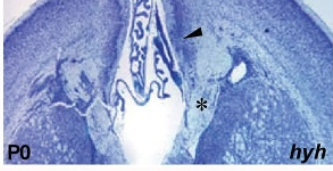

Reelin/Hoechst
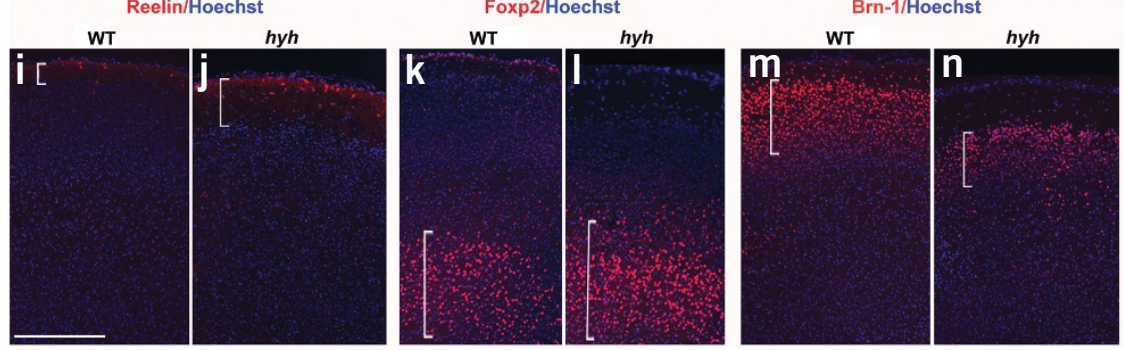

between the two hemispheres (arrow). The corpus callosum (CC) did not form, instead producing Probst bundles composed of cortical axons arrested in their normal midline growth (asterisk). There were multiple laminar disruptions of cingulate and hippocampal cortex (arrowhead). (i-n) Neuronal cell types specific to particular stages of neurogenesis and to particular cell layers of the cortex were examined by immunohistochemistry for Reelin (i,j), Foxp2 (k,I) and Brn-1 $(\mathbf{m}, \mathbf{n})$ in coronal sections of the anterior cerebral cortex after the completion of neurogenesis at P3. Cajal-Retzius cells, among the earliest born neurons of the cortex, were detected by the CR50 monoclonal antibody against Reelin ${ }^{30}$. The number of Reelin-positive neurons was roughly two times higher ( 15 cells versus 31 cells per $200 \mu \mathrm{m}$ thickness of cortex) in hyh embryos (j) than in wild-type embryos (i). The marginal zone, the most superficial and cell-sparse layer of the cerebral cortex that contains the Cajal-Retzius cells, was proportionally about 1.9 times thicker in hyh embryos (brackets in $\mathbf{i}, \mathbf{j}$ ). Foxp2 immunostaining is used to detect the early-born layer VI neurons (k,I). Foxp2 staining showed a greater density of layer VI neurons in cortex of hyh embryos compared with wild-type embryos ( 112 cells versus 73 cells per $100 \mu \mathrm{m}^{2}$ ). Furthermore, Foxp2 staining showed a 1.4 -fold expansion of the vertical extent of the cortex that contains Foxp2-positive cells in hyh embryos compared with wild-type embryos (brackets). Foxp2-positive cells occupied 53\% of the total cortical thickness (as defined by the distance from the pial surface to the lower limit of Foxp2 immunoreactivity) in hyh mutants compared with $39 \%$ in wildtype embryos. In contrast, Brn-1 immunostaining for the later-born neurons of layers II-IV showed fewer layer II-IV neurons and a corresponding decrease in the thickness of layers II-IV from $24 \%$ of the cortical thickness (as defined above) in wild-type embryos (m) to $17 \%$ in $h y h$ (n) this was confirmed with antisera to Cux-1 (data not shown). Scale bar, $100 \mu \mathrm{m}$. 
that regulates asymmetrical cell fates in the fly sensory organ ${ }^{16}$, was also disrupted (Fig. 5c,d). Immunoreactivity to $\beta$-catenin, which binds the cytoplasmic tail of E-cadherin, was much more diffuse in $h y h$ mutants, without clear localization to adherens junctions (Fig. $\mathbf{5 e}, \mathbf{f})$. Intensity of apical cytoplasmic $\beta$-catenin staining in neuroepithelial cells was also lower. Distributions of other apical proteins, including aPKC and INADL (ref. 17; Fig. 5g-j), were also abnormal in neuroepithelium of hyh mutants. These disturbances of junctional complexes are presumed to lead to the denudation of the apical epithelium ${ }^{18}$. In flies and worms, the apical complex proteins help localize cell fate determinants, such as Numb and Miranda, but there are redundant mechanisms for Numb localization ${ }^{19,20}$. Immunoreactivity to mouse $\mathrm{Numb}^{21}$ in hyh mutants showed apical localization that was indistinguishable from that of normal mice (data not shown), suggesting that Numb localization may be controlled by redundant pathways in mice as well. Given the roles for $\beta$ catenin, adherens junctions and other apical proteins in control of cell fate, however, disruption of their normal localization could cause defects in cortical cell fate and growth of hyh mutants.

Overall vesicle morphology was normal in neuroepithelial cells of $h y$, but apical vesicle localization was severely abnormal. Localization and structure of the Golgi apparatus in embryonic neuroepithelial cells and adult neurons was normal as examined by electron microscopy or by GM130 immunostaining (data not shown). In contrast, the localization of Vamp7 (also known as TI-VAMP), a vesicle SNARE involved in apical membrane transport in epithelial cells ${ }^{22,23}$ and neurons $\mathrm{s}^{24,25}$, was strongly apical in normal neuroepithelial cells and profoundly disrupted in hyh mutants (Fig. 5k,l), suggesting that partial loss of $\alpha$ Snap disrupted its apical targeting function without disrupting all its cellular functions. The widespread expression of $\alpha$ Snap in the developing embryo (Supplementary Fig. 3 and

Figure 4 Precocious neurogenesis and progenitor depletion in cortex of hyh mutants. Immunostaining for Nestin, a marker of neural progenitors (red); CSPG, a marker of early-born preplate neurons (red); and Doublecortin (Dcx), a marker of early postmitotic neurons (red), was used to compare the population of neural progenitors and differentiated neurons. Nuclei are marked by Hoechst staining (blue). (a-d') At E12.5, the cortex of wild-type embryos contained a dense population of Nestin-immunoreactive cells throughout its thickness $\left(\mathbf{a}, \mathbf{a}^{\prime}\right)$, whereas the cortex of hyh embryos contained markedly fewer Nestin-positive cells (b, $\left.\mathbf{b}^{\prime}\right)$. At E14.5, immunostaining with antibody to Nestin was intense throughout the wildtype cortex and labeled cells with typical radial glial morphology $\left(\mathbf{c}, \mathbf{c}^{\prime}\right)$ but was markedly reduced in hyh cortex $\left(\mathbf{d}, \mathbf{d}^{\prime}\right)$. This was confirmed with RC-2 staining of radial glial cells (data not shown). (e-f') Immunostaining for CSPG, a marker of preplate cells, the earliest-born population of neurons, at E14.5 showed more CSPG-positive neurons in hyh embryos $\left(\mathbf{f}, \mathbf{f}^{\prime}\right)$ than in wild-type $\left(\mathbf{e}, \mathbf{e}^{\prime}\right)$. CSPG staining also showed that preplate splitting by cortical plate neurons (CP) occurred normally in the cortex of hyh embryos despite the expansion of the cell population. (g-r) Dcx immunostaining labeled a broader area and a greater number of cells in the cortex of hyh embryos (i, $\mathbf{m}, \mathbf{q})$ than wild-type embryos $(\mathbf{g}, \mathbf{k}, \mathbf{0})$ during early (E12.5 and E14.5) and mid-neurogenesis (E16.5). At E12.5, Dcx-positive neurons formed a narrow superficial layer of the cortex in wild-type embryos $(\mathbf{g}, \mathbf{h})$ but were more numerous and composed a greater proportion of the cortical thickness in hyh embryos (i,j). At E14.5, Dcx-positive cells again occupied a larger extent of cortex in hyh embryos (n) versus wild-type embryos (I), and the number and density of Dcx-positive cells was greater in hyh mutants. At E16.5, the cortex of hyh embryos ( $\mathbf{r}$ ) was more densely packed with Dcx-positive cells than that of wild-type embryos (p), and the cortex contained only a narrow margin of Dcx-negative cells, representing a diminished ventricular zone, in hyh embryos relative to wild-type. Similar patterns were seen with immunoreactivity to TuJ1 and Vamp2, which also mark postmitotic neurons (data not shown).

Supplementary Methods online) also suggested that the hyh mutation uncovers a dose-related role in apical trafficking.

The hyh mutation suggests that $\alpha$ Snap-mediated vesicle trafficking regulates cell fate by regulating the key choice between forming proliferative and postmitotic daughter cells. The overproduced early-born neurons seem to adopt specific neuronal fates appropriate for their birth date, as observed in other systems ${ }^{26}$. Regulation of cell fate by $\alpha$ Snap may affect other organs, but previous analysis has not shown hypoplasia of other organs ${ }^{1}$ in $h y h$ mice, perhaps because other tissues regulate size postnatally, whereas the postnatal brain contains largely postmitotic neurons. Other genes that affect the size of the central nervous system in humans commonly do not affect other organs, even when they are ubiquitously expressed ${ }^{27,28}$. Other tissues may also have more redundancy for $\alpha$ Snap function during embryogenesis, through $\gamma$ Snap, or the remaining mutant $\alpha$ Snap in hyh mice may suffice for membrane trafficking in other tissues. Although the potential role of $\alpha$ Snap in apical trafficking has previously been controversial ${ }^{29}$, analysis of the hyh mutant supports this role and provides an opportunity to examine the role of apical vesicle trafficking in other aspects of neural development. The hyh mutant has additional defects in cerebellar development, neuronal migration and axonal pathfinding (data not shown), suggesting roles for apical trafficking in these processes.

\section{METHODS}

Mice. We obtained mice from The Jackson Laboratory, where the hyh mutation originally arose in the $\mathrm{C} 57 \mathrm{BL} / 10 \mathrm{~J}$ inbred strain and was subsequently placed on a $\mathrm{B} 6 \mathrm{C} 3 \mathrm{Fe}-a / a(\mathrm{C} 57 \mathrm{BL} / 6 \mathrm{~J}$ female $\times \mathrm{C} 3 \mathrm{HeB} / \mathrm{FeJ}-a / a$ male $)$ hybrid background ${ }^{1}$. We generated backcross and intercross progeny and genotyped them as previously described ${ }^{2}$. Mice were housed and handled in accordance with protocols approved by the Institutional Animal Care and Use Committee of Harvard Medical School.

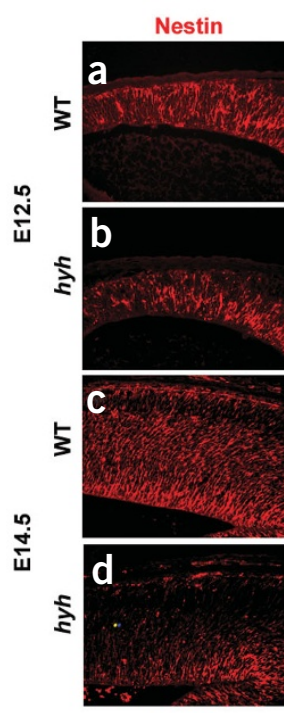

CSPG

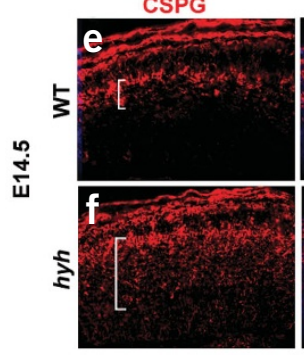

Nestin/Hoechst
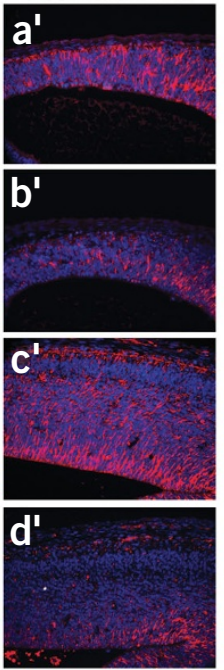

CSPG/Hoechst

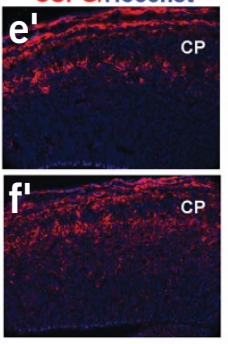

DCX
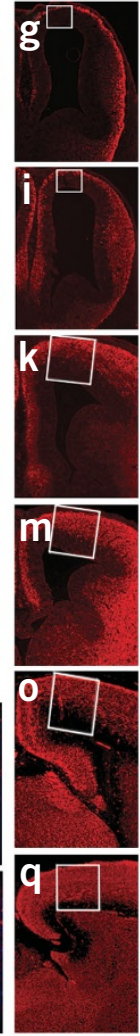

DCX
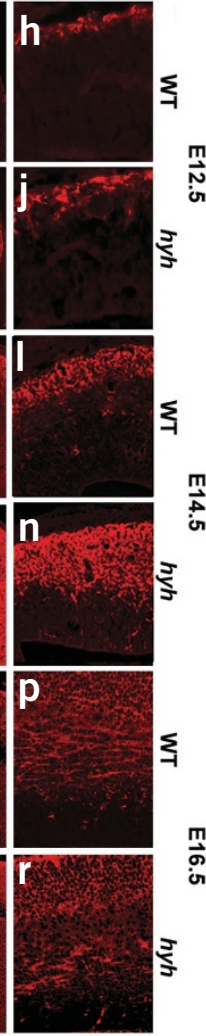
Identification of genes in the $h y h$ candidate interval. We isolated BACs from the CITB mouse BAC library (Research Genetics) and RPCI-23 mouse BAC library (Research Genetics) and identified genes by shotgun sequencing of BACs as previously described and use of publicly available mouse and human genome sequence data ${ }^{2}$. We confirmed the presence of these genes in the contig spanning the $h y$ candidate interval by PCR.

New polymorphic STSs. The previously described markers Mrg2, Kptn and 293M16.R (ref. 2) and the newly designed STSs in Napa and Kiaa1087 contained single strand conformation polymorphisms (SSCPs) between the mouse strains $\mathrm{C} 57 \mathrm{~B} 10 / \mathrm{J}$ and $\mathrm{C} 3 \mathrm{HeB} / \mathrm{FeJ}-a / a$. The newly designed STSs contained in BACs 93K16, 93K16.F and 93K16.2 contained simple sequence length polymorphisms (SSLPs) between these mouse strains. Primer sequences are available on request. We analyzed SSCPs and SSLPs as previously described ${ }^{2}$.

Northern-blot analysis. We extracted total RNA using TRIzol (Invitrogen), denatured $10 \mathrm{mg}$ of each sample, separated it by electrophoresis in a $1 \%$ agarose formaldehyde gel and transferred products to a Hybond-N nylon membrane (Amersham Pharmacia Biotech). Blots were hybridized to ${ }^{32} \mathrm{P}$-labeled probes in ExpressHyb (BD Biosciences Clontech) at $65^{\circ} \mathrm{C}$ for $1 \mathrm{~h}$. We rinsed blots several times in $2 \times$ saline sodium citrate (SSC) and $0.1 \%$ SDS at room temperature, washed them twice for $20 \mathrm{~min}$ each in $0.1 \times$ SSC and $0.1 \%$ SDS at $58{ }^{\circ} \mathrm{C}$ and exposed them to film overnight at $-80^{\circ} \mathrm{C}$ with an intensifying screen. We generated the Napa probe by PCR using primers to the $3^{\prime}$ untranslated region. Primer sequences are available on request.

RT-PCR and sequencing of Napa. We carried out reverse transcription using SUPERSCRIPTII Reverse Transcriptase according to manufacturer's specifications (Invitrogen). We designed two overlapping sets of PCR primers to amplify the entire coding sequence and a portion of the $3^{\prime}$ untranslated region of Napa. Primer sequences available on request. We amplified Napa cDNAs from first-strand cDNA from $h y h / h y h$ mice and wild-type littermates. We used $\sim 50 \mathrm{ng}$ as template for sequencing using the ABI Big Dye Cycle Sequencing Kit and ABI 377 Sequencer (Applied Biosystems). We confirmed that the identified nucleotide change was present in $h y h / h y h$ genomic DNA and absent from that of wild-type littermates, C57BL/10J, $\mathrm{C} 3 \mathrm{HeB} / \mathrm{FeJ}-a / a$ and $\mathrm{C} 57 \mathrm{BL} / 6 \mathrm{~J}$ mice by PCR and sequencing using primers specific to exon 4 of Napa. Primer sequences are available on request.

Figure 5 Abnormalities of the ventricular neuroepithelium in hyh mutants. Immunostaining for apically and apical-laterally localized proteins F-actin, $\beta$-catenin, E-cadherin, Numb and aPKC in coronal sections of cerebral cortex at E14.5 showed defects of protein localization in neuroepithelium of hyh embryos. (a,b) Rhodamine-phalloidin stain visualizes the actin scaffold and adherens junctions, shown as evenly spaced actin-rich structures in wild-type ventricular cells (a). In hyh embryos (b), phalloidin staining in ventricular cells was more diffuse, losing its evenly spaced distribution, and, in some areas, was ectopically localized away from the apical surface (arrows). (c,d) Immunostaining for adherens junction component E-cadherin showed enrichment of E-cadherin at the apicallateral surface of the neuroepithelium in wild-type embryos and much more diffuse distribution in hyh embryos, with greater staining of the basolateral surfaces of epithelial cells than in normal and a loss of the normal concentration of immunoreactivity at the adherens junction. Focal areas of increased immunoreactivity outside the ventricular surface probably represent ectopic adherens junctions. (e,f) $\beta$-catenin immunoreactivity also showed less overall immunoreactivity and substantial loss of the normal pattern of intense accumulation at the apical-lateral adherens junctions, with appearance of abnormal aggregates in the cytoplasm (arrows), indicating impairment of directed apical-lateral targeting. (g,h) Immunoreactivity for aPKC, a component of the Par3/Par6 complex that normally concentrates apically, was fainter and poorly localized in hyh embryos. $(\mathbf{i}, \mathbf{j})$ INADL immunoreactivity was similarly reduced in its normal apical concentration. (k,I) Punctate immunoreactivity to Vamp7 (arrows), a component of the apical vesicle transport machinery, was less abundant and had poor apical localization. Scale bars, $3 \mu \mathrm{m}$.
Western blotting. We dissected brains from E14.5 embryos in ice-cold phosphate-buffered saline (PBS), froze them immediately in liquid nitrogen and stored them at $-80{ }^{\circ} \mathrm{C}$ until use. We homogenized the tissue in $1 \mathrm{ml}$ of $95^{\circ} \mathrm{C} 2 \times$ sample buffer and passed it through a 1-cc insulin syringe to shear genomic DNA. We separated equal amounts of each lysate on a 10\% SDS-PAGE gel and transferred them to Immobilon-P membranes (Millipore). We blocked the membranes in 5\% milk and Tris-buffered saline Tween-20 (TBST) for at least $30 \mathrm{~min}$, incubated them with $\alpha$ Snap monoclonal antibody (Exalpha Biologicals) at 1:1,000 dilution in blocking solution for $1 \mathrm{~h}$ at room temperature with agitation, washed them in TBST three times for $10 \mathrm{~min}$ each, incubated them with secondary antibody in blocking solution for $1 \mathrm{~h}$ at room temperature and finally washed them three times for 15 min each in TBST. We developed blots using LumiGlo substrate (Kirkegaard and Perry Laboratories) according to manufacturer's instructions and exposed them to film. We then stripped the blots and reprobed them with antibody to tubulin (Sigma) diluted to $1: 1,000$ as a loading control.

BAC complementation. We obtained BAC clones from Research Genetics and isolated BAC DNA using the Nucleobond BAC Maxi Kit (BD Biosciences Clontech). We injected DNA at a concentration of $1 \mathrm{ng} \mathrm{ml}^{-1}$ into fertilized eggs from FVB mice to generate transgenic mice at the Children's Hospital transgenic services core facility. We implanted fertilized eggs into foster mothers and identified transgenic founders by PCR detection of BAC vector sequence. All subsequent housing and breeding of transgenic lines took place at Charles River Laboratories. Transgenic founders were bred to hyh heterozygotes and the resulting progeny were genotyped for the transgene and at 116J6.1 and 293M16.R or D7Mit56 to determine the genotype at the hyh locus. Those determined to be heterozygous at the hyh locus and positive for the transgene were
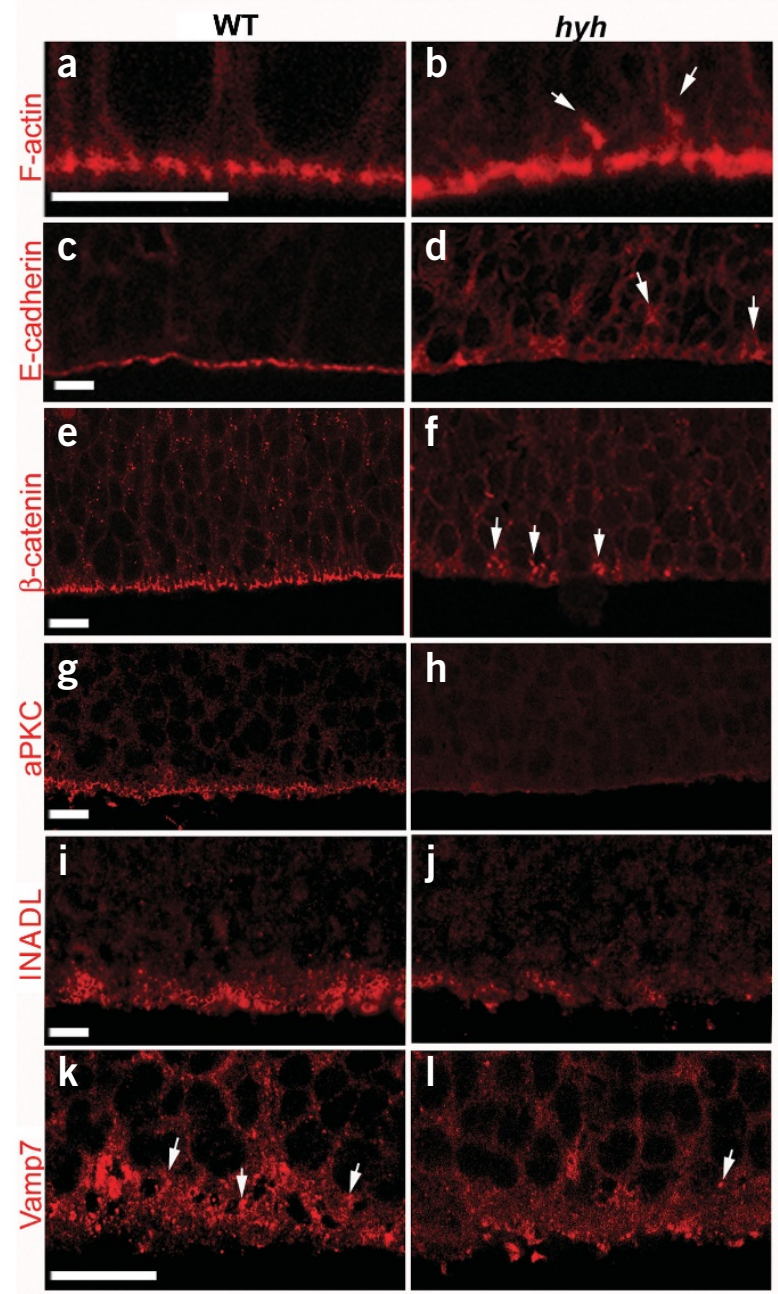
intercrossed or crossed to hyh heterozygotes without the transgene. Resulting progeny were again genotyped as above; mice of interest were perfused with $10 \%$ neutral-buffered formalin and their brains were collected at Charles River Laboratories. In several cases of failure of rescue or incomplete penetrance of the hyh phenotype, SSCP analysis for the Napa mutation showed absence and presence, respectively, of a wild-type allele of Napa that was segregating independently of the marker used to genotype for the transgene.

In vitro comparison of wild-type and M105I $\alpha$ Snap. We made $\mathrm{His}_{6}$-tagged M105I $\alpha$ Snap by Quikchange site-directed mutagenesis of bovine $\alpha$ Snap in pET28a (ref. 3). We expressed, purified and quantified wild-type and mutant $\alpha$ Snap and SNARE proteins as described ${ }^{3}$. We obtained CD spectra in $20 \mathrm{mM}$ Tris, $100 \mathrm{mM} \mathrm{NaCl}$ using a JASCO J600 spectropolarimeter as described ${ }^{3}$. We monitored $\alpha$ Snap binding to synaptic SNARE complex (comprised of syntaxin1a(1-265)-cyan fluorescent protein (CFP), SNAP-25b and synaptobrevin II (1-96)) by competition between untagged and yellow fluorescent protein (YFP)-tagged $\alpha$ Snap. YFP- $\alpha$ Snap $(2 \mu \mathrm{M})$ bound to CFP-tagged SNARE complex $(50 \mathrm{nM})$ led to FRET, which we monitored as a quenching of the syntaxin CFP signal. We added untagged $\alpha$ Snap (wild-type or M105I; $8 \mu \mathrm{M}$ ) as competitor and decreased the FRET. We measured fluorescence in a SPEX Fluorolog-3 fluorimeter with excitation at $425 \mathrm{~nm}$, emission at $472 \mathrm{~nm}$ and slits at $3.5 \mathrm{~nm}$. We monitored SNARE complex disassembly by NSF in the presence of wildtype or M105I $\alpha$ Snap essentially as described ${ }^{3}$, taking advantage of FRET between syntaxin1a(1-265)-CFP and synaptobrevin II(1-96)-YFP when the proteins are bound to each other in a SNARE complex. We preincubated reactions containing SNARE complex $(50 \mathrm{nM})$ and $\alpha$ Snap $(0.8 \mu \mathrm{M})$ in $500 \mu \mathrm{l}$ buffer (30 mM HEPES, pH 7.6, $100 \mathrm{mM}$ glutamic acid, $10 \mathrm{mM} \mathrm{MgCl}$, $2 \mathrm{mM}$ ATP, $0.1 \%$ Triton $\mathrm{X}-100,1 \mathrm{mg} \mathrm{ml}^{-1}$ bovine serum albumin) at $30{ }^{\circ} \mathrm{C}$ before initiating a disassembly reaction by adding NSF $(1.5 \mu \mathrm{g})$. CFP fluorescence was followed as a function of time, with excitation at $434 \mathrm{~nm}$ and emission at $472 \mathrm{~nm}$.

Histology. We collected embryonic tissue in ice-cold PBS and fixed it by immersion in $4 \%$ paraformaldehyde in PBS at $4{ }^{\circ} \mathrm{C}$ overnight. We collected adult tissue after intracardiac perfusion with PBS and then $4 \%$ paraformaldehyde in PBS. We postfixed the tissue in $4 \%$ paraformaldehyde in PBS at $4{ }^{\circ} \mathrm{C}$ overnight, embedded it in paraffin, cut it into 5-mm sections and mounted sections to slides. We removed the paraffin from sections with xylene, rehydrated the sections through an ethanol series into water and stained them in cresyl violet.

Immunohistochemistry. We prepared 5-mm sections as above, removed the paraffin and rehydrated sections through an ethanol series into distilled water. After antigen retrieval using Antigen Unmasking Solution (Vector Laboratories) according to manufacturer's instructions, we rinsed sections in PBS and blocked them with $5 \%$ goat serum in $0.3 \%$ Triton X-100 in PBS for 30 min at room temperature. We applied the following antibodies to sections and incubated them at room temperature for $1-3 \mathrm{~h}$ : $\beta$-catenin (1:100, BD Biosciences), E-cadherin (1:500, Transduction Laboratories), Numb (1:200, gift from W. Zhong, Yale University), Dcx ${ }^{9}$ (1:500), Nestin (1:500, Developmental Studies Hybridoma Bank), Foxp2 (1:500, gift from E. Morrissey, University of Pennsylvania), aPKC (1:500, BD Biosciences), Cux1 (1:200, gift from J. Cunningham, Brigham and Women's Hospital and Harvard Medical School), RC-2 (1:200, Developmental Studies Hybridoma Bank), phosphorylated histone H3 (1:500, Upstate), Vamp2 (1:500, Sysy), GFAP (1:500, Sigma), GM130 (1:500, Transduction Laboratories), Brn-1 (1:200, Santa Cruz), Vamp7 (1:250, gift from T. Galli, Institut du Fer-àMoulin, Paris), INADL (1:500) or CR50 antibody (1:30, gift from M. Ogawa, RIKEN, Japan). We raised an antiserum to INADL by synthesizing a peptide identical to the N-terminal 14 amino acids (TEESDSGIKNDLC) of INADL and immunizing rabbits using standard methods. We washed sections in $0.3 \%$ Triton X-100 in PBS three times for 5 min each. We applied a 1:500 dilution of biotinylated goat antibody to rabbit IgG (Vector Laboratories) or a 1:150 dilution of biotinylated goat antibody to mouse IgG (Jackson ImmunoResearch Laboratories) in blocking solution for $1-3 \mathrm{~h}$ at room temperature. We washed sections as described above, incubated them with streptavidin-conjugated $\mathrm{Cy} 3$ diluted 1:500 in blocking solution for $1 \mathrm{~h}$ at room temperature, washed them again as described above, stained them with Hoechst 33342 diluted 1:1,000 in PBS, rinsed them in PBS and mounted them in VECTASHIELD mounting media (Vector Laboratories).
URLs. Analysis of mouse genome draft sequence is available at http://www. ncbi.nlm.nih.gov. Human genome sequence of the homologous region of human Chromosome 19q13 is available at http://genome.ucsc.edu.

Note: Supplementary information is available on the Nature Genetics website.

\section{ACKNOWLEDGMENTS}

We thank M. Davisson and H. Sweet for their help and collaboration on early genetic mapping; T. Thompson for assistance with transgenic mouse production; U. Berger for in situ hybridizations; S.-H. Cho for help with confocal imaging; J. Lauer for help with SNARE complex assays; the Developmental Studies Hybridoma Bank for antibodies; W. Zhong, T. Galli, M. Ogawa, J. Cunningham and E. Morrissey for providing antisera; and K. Allen for use of several slides pictured in Figure 3 and for her ongoing enthusiasm and support of this project. T.H.C. was supported by the US National Institutes of Health Medical Scientist Training Program and the Adams/Quan Fellowship. S.K. is supported by a Helen Hay Whitney Postdoctoral fellowship. Transgenic work was supported by the Mental Retardation Research Center at Children's Hospital, Boston. P.I.H. and C.A.W. were supported by grants from the US National Institute of Neurological Disease and Stroke. P.I.H. is a W.M. Keck Foundation Distinguished Young Scholar. C.A.W. is an Investigator of the Howard Hughes Medical Institute.

\section{COMPETING INTERESTS STATEMENT}

The authors declare that they have no competing financial interests.

Received 23 December 2003; accepted 20 January 2004

Published online at http://www.nature.com/naturegenetics/

1. Bronson, R.T. \& Lane, P.W. Hydrocephalus with hop gait (hyh): a new mutation on chromosome 7 in the mouse. Brain Res. Dev. Brain Res. 54, 131-136 (1990).

2. Chae, T.H., Allen, K.M., Davisson, M.T., Sweet, H.O. \& Walsh, C.A. Mapping of the mouse hyh gene to a YAC/BAC contig on proximal Chromosome 7. Mamm Genome 13, 239-244 (2002).

3. Marz, K.E., Lauer, J.M. \& Hanson, P.I. Defining the SNARE complex binding surface of alpha-SNAP: implications for SNARE complex disassembly. J. Biol. Chem. 278, 27000-27008 (2003).

4. Piano, F., Schetter, A.J., Mangone, M., Stein, L. \& Kemphues, K.J. RNAi analysis of genes expressed in the ovary of Caenorhabditis elegans. Curr. Biol. 10, 1619-1622 (2000).

5. D'Arcangelo, G. et al. Reelin is a secreted glycoprotein recognized by the CR-50 monoclonal antibody. J. Neurosci. 17, 23-31 (1997).

6. Ferland, R.J., Cherry, T.J., Preware, P.O., Morrisey, E.E. \& Walsh, C.A Characterization of Foxp2 and Foxp1 mRNA and protein in the developing and mature brain. J. Comp. Neurol. 460, 266-279 (2003).

7. McEvilly, R.J., de Diaz, M.O., Schonemann, M.D., Hooshmand, F. \& Rosenfeld, M.G. Transcriptional regulation of cortical neuron migration by POU domain factors. Science 295, 1528-1532 (2002)

8. Pearlman, A.L. \& Sheppard, A.M. Extracellular matrix in early cortical development. Prog. Brain Res. 108, 117-134 (1996).

9. Gleeson, J.G., Lin, P.T., Flanagan, L.A. \& Walsh, C.A. Doublecortin is a microtubuleassociated protein and is expressed widely by migrating neurons. Neuron 23, 257-271 (1999).

10. Takahashi, T., Nowakowski, R.S. \& Caviness, V.S., Jr. Mode of cell proliferation in the developing mouse neocortex. Proc. Natl. Acad. Sci. USA 91, 375-379 (1994)

11. Caviness, V.S. Jr. \& Takahashi, T. Proliferative events in the cerebral ventricular zone. Brain Dev. 17, 159-163 (1995).

12. Caviness, V.S. Jr., Takahashi, T. \& Nowakowski, R.S. Numbers, time and neocortical neuronogenesis: a general developmental and evolutionary model. Trends Neurosci. 18, 379-383 (1995).

13. Takahashi, T., Nowakowski, R.S. \& Caviness, V.S. Jr. The cell cycle of the pseudostratified ventricular epithelium of the embryonic murine cerebral wall. J. Neurosci. 15, 6046-6057 (1995).

14. Clary, D.O., Griff, I.C. \& Rothman, J.E. SNAPs, a family of NSF attachment proteins involved in intracellular membrane fusion in animals and yeast. Cel/ 61, 709-721 (1990).

15. Puschel, A.W., O'Connor, V. \& Betz, H. The N-ethylmaleimide-sensitive fusion protein (NSF) is preferentially expressed in the nervous system. FEBS Lett. 347, 55-58 (1994)

16. Le Borgne, R., Bellaiche, Y. \& Schweisguth, F. Drosophila E-cadherin regulates the orientation of asymmetric cell division in the sensory organ lineage. Curr. Biol. 12, 95-104 (2002).

17. Hurd, T.W., Gao, L., Roh, M.H., Macara, I.G. \& Margolis, B. Direct interaction of two polarity complexes implicated in epithelial tight junction assembly. Nat. Cell Biol. 5, 137-142 (2003).

18. Jimenez, A.J. et al. A programmed ependymal denudation precedes congenital hydrocephalus in the hyh mutant mouse. J. Neuropathol. Exp. Neurol. 60, 1105-1119 (2001). 
19. Cai, Y., Yu, F., Lin, S., Chia, W. \& Yang, X. Apical complex genes control mitotic spindle geometry and relative size of daughter cells in Drosophila neuroblast and $\mathrm{pl}$ asymmetric divisions. Cell 112, 51-62 (2003).

20. Yu, F., Cai, Y., Kaushik, R., Yang, X. \& Chia, W. Distinct roles of Galphai and Gbeta13F subunits of the heterotrimeric $G$ protein complex in the mediation of Drosophila neuroblast asymmetric divisions. J. Cell Biol. 162, 623-633 (2003).

21. Zhong, W., Feder, J.N., Jiang, M.M., Jan, L.Y. \& Jan, Y.N. Asymmetric localization of a mammalian numb homolog during mouse cortical neurogenesis. Neuron 17, 43-53 (1996).

22. Bogdanovic, A. et al. Syntaxin 7, syntaxin 8, Vti1 and VAMP7 (vesicle-associated membrane protein 7) form an active SNARE complex for early macropinocytic compartment fusion in Dictyostelium discoideum. Biochem. J. 368, 29-39 (2002).

23. Lafont, F. et al. Raft association of SNAP receptors acting in apical trafficking in Madin-Darby canine kidney cells. Proc. Natl. Acad. Sci. USA 96, 3734-3738 (1999).

24. Coco, S. et al. Subcellular localization of tetanus neurotoxin-insensitive vesicle-associated membrane protein (VAMP)/VAMP7 in neuronal cells: evidence for a novel membrane compartment. J. Neurosci. 19, 9803-9812 (1999).
25. Martinez-Arca, S., Alberts, P., Zahraoui, A., Louvard, D. \& Galli, T. Role of tetanus neurotoxin insensitive vesicle-associated membrane protein (TI-VAMP) in vesicular transport mediating neurite outgrowth. J. Cell Biol. 149, 889-900 (2000).

26. Reh, T.A. \& Kljavin, I.J. Age of differentiation determines rat retinal germinal cell phenotype: induction of differentiation by dissociation. J. Neurosci. 9, 4179-4189 (1989).

27. Sheen, V.L. et al. Mutations in ARFGEF2 implicate vesicle trafficking in neural progenitor proliferation and migration in the human cerebral cortex. Nat. Genet. 36, 69-76 (2004).

28. Kolehmainen, J. et al. Cohen Syndrome is caused by mutations in a novel gene, $\mathrm{COH} 1$, encoding a transmembrane protein with a presumed role in vesicle-mediated sorting and intracellular protein transport. Am. J. Hum. Genet. 72, 1359-1369 (2003).

29. Low, S.H. et al. The SNARE machinery is involved in apical plasma membrane trafficking in MDCK cells. J. Cell Biol. 141, 1503-1513 (1998)

30. Ogawa, M. et al. The reeler gene-associated antigen on Cajal-Retzius neurons is a crucial molecule for laminar organization of cortical neurons. Neuron 14, 899-912 (1995) 